\title{
Bogomolov multiplier, double class-preserving automorphisms and modular invariants for orbifolds
}

\author{
Alexei Davydov
}

July 18, 2021

Department of Mathematics, Ohio University, Athens, OH 45701, USA

\begin{abstract}
We describe the group $A u t_{b r}^{1}(\mathcal{Z}(G))$ of braided tensor autoequivalences of the Drinfeld centre of a finite group $G$ isomorphic to the identity functor (just as a functor). We prove that the semi-direct product $O u t_{2-c l}(G) \ltimes B(G)$ of the group of double class preserving automorphisms and the Bogomolov multiplier of $G$ is a subgroup of $A u t_{b r}^{1}(\mathcal{Z}(G))$. An automorphism of $G$ is double class preserving if it preserves conjugacy classes of pairs of commuting elements in G. The Bogomolov multiplier $B(G)$ is the subgroup of its Schur multiplier $H^{2}\left(G, k^{*}\right)$ of classes vanishing on abelian subgroups of $G$. We show that elements of $A u t_{b r}^{1}(\mathcal{Z}(G))$ give rise to different realisations of the charge conjugation modular invariant for $G$-orbifolds of holomorphic conformal field theories.
\end{abstract}

\section{Contents}

1 Introduction 1

2 Group-theoretical braided fusion categories and their autoequivalences 3

2.1 Soft tensor autoequivalences . . . . . . . . . . . . . . . . . . 3

2.2 Soft tensor autoequivalences of graded vector spaces . . . . . . . . . . . . . . . . . . 4

2.3 Soft braided tensor autoequivalences of categories of representations . . . . . . . . 6

2.4 Soft braided tensor autoequivalences of Drinfeld centres of finite groups . . . . . . 7

3 Examples 10

3.1 Bogomolov multipliers . . . . . . . . . . . . . . . . . . . . . . 10

3.2 Double class-preserving automorphisms . . . . . . . . . . . . . . . . 11

4 Applications 11

4.1 Lagrangian algebras of braided autoequivalences . . . . . . . . . . . . . . . 11

4.2 Holomorphic permutation orbifolds . . . . . . . . . . . . . . . . . . 12

\section{Introduction}

The aim of this note is to construct examples of different rational conformal field theories with the same chiral algebras and the same (charge conjugation) modular invariant.

The state space of a (2-dimesional) conformal field theory comes equipped with amplitudes associated to a finite collection of fields inserted into a surface [12, 33. Fields, whose amplitudes depend (anti-)holomorphically on insertion points, form what is known as (anti-)chiral algebra of the CFT. The state space is naturally a representation of the product of the chiral and anti-chiral algebras. A conformal field theory is rational if the state space is a finite sum of tensor products of irreducible representations of chiral and anti-chiral algebras. The matrix of multiplicities of 
irreducible representations in the decomposition of the state space is called the modular invariant of the RCFT. The simplest case (the so-called Cardy case) is the case of the charge conjugation modular invariant, which assumes that chiral and anti-chiral algebras coincide.

The name modular invariant comes from the fact that the matrix of multiplicities is invariant with respect to the modular group action on characters. This fact was used in 4 to classify modular invariants for affine $\operatorname{sl}(2)$ rational conformal field theories. This paper started an activity aimed at classifying possible modular invariants for various conformal field theories. It took some time to realise that not all modular invariants correspond to conformal field theories 24, 17. In this paper we show that there are different rational conformal field theories with the same (charge conjugation) modular invariant. Thus although being a convenient numerical invariants of a rational conformal field theory modular invariants are far from being complete.

An adequate description of rational conformal field theories was obtained relatively recently (see [23] and references therein). Mathematical axiomatisation of chiral algebras in CFTs is the notion of vertex operator algebra [3, 16, 28. A vertex operator algebra is called rational if it is a chiral algebra of a RCFT, in particular its category of modules is semi-simple. In this case the category of modules has more structure (see [25] and references therein), it is the so-called modular category. This type of tensor categories was first studied in physics 33 and then axiomatised mathematically in 39. The state space of a RCFT corresponds to a special commutative algebra in the product or categories of modules of chiral and anti-chiral algebras (see [18, 19, 20, 21, 22, 15, 26. and references therein). These special class of commutative algebras in braided tensor categories is known as Lagrangian algebras [9. The modular invariant is just the class of this Lagrangian algebra in the Grothendieck ring.

Here we give examples of Lagrangian algebras with the charge conjugation modular invariant. It is straightforward to see that such algebras should correspond to braided tensor autoequivalences of the category of representations of one of the chiral algebra. A braided tensor autoequivalence corresponds to the charge conjugation modular invariant if it does not permute (isomorphism classes of) simple objects. We call such braided tensor autoequivalence soft. We provide examples of modular categories with soft braided tensor autoequivalences.

Our examples come from permutation orbifolds of holomorphic conformal field theories (CFTs whose state space is an irreducible module over the chiral algebras). It is argued in [30] (see also [35]) that the modular category of the $G$-orbifold of a holomorphic conformal field theory is the so called Drinfeld (or monoidal) centre $\mathcal{Z}(G, \alpha)$, where $\alpha$ is a 3-cocycle of the group $G$. It is also known that the cocycle $\alpha$ is trivial for permutation orbifolds (orbifolds where the group $G$ is a subgroup of the symmetric group permuting copies in a tensor power of a holomorphic theory). The assumption crucial for the arguments of [30] is the existence of twisted sectors. This assumption is known to be true for permutation orbifolds [1]

Clearly the property of a braided tensor autoequivalence to preserve the isomorphism classes of simple objects is stable under the composition of tensor autoequivalences. Thus the (tensor) isomorphism classes of soft braided tensor autoequivalences of a category $\mathcal{C}$ form a group $A u t_{b r}^{1}(\mathcal{C})$. In this paper we describe the group $A u t_{b r}^{1} t(\mathcal{Z}(G))$ as a semi-direct product:

$$
A u t_{b r}^{1}(\mathcal{Z}(G)) \simeq \text { Out }_{2-c l}(G) \ltimes B(G) .
$$

The subgroup $B(G)$ is the so-called Bogomolov multiplier of a finite group $G$. It is a subgroup of the Schur multiplier $H^{2}\left(G, k^{*}\right)$ defined as the kernel of the restriction map

$$
B(G)=\operatorname{ker}\left(H^{2}\left(G, k^{*}\right) \stackrel{\text { res }}{\longrightarrow} \bigoplus_{A \subset G} H^{2}\left(A, k^{*}\right)\right)
$$

where the direct sum is taken over all abelian subgroups of $G$.

The group Out $_{2-c l}(G)$ is a subgroup of the group $\operatorname{Out}(G)$ of the outer automorphisms of $G$ (classes of automorphisms modulo inner automorphisms). The subgroup $\mathrm{Out}_{2-c l}(G)$ consists of outer automorphisms preserving conjugacy classes of pairs of commuting elements in $G$. The action of $\mathrm{Out}_{2-c l}(G)$ on $B(G)$ comes from the natural action of $\mathrm{Out}(G)$ on $H^{2}\left(G, k^{*}\right)$. 
The Bogomolov multiplier is an important invariant of birational geometry [2]. Recent activity produced examples of finite groups with non-trivial Bogomolov multiplier (see for example [5, 27]). Finite groups with non-trivial $\mathrm{Out}_{2-c l}(G)$ were constructed in 38 .

The paper is organised as follows. We start by defining soft braided tensor autoequivalences and collect some of their basic properties in section 2.1. Then we recall necessary facts about tensor autoequivalences of the categories of group-graded vector spaces and of representations of groups (section 2.2, 2.3). We proceed by proving our main result - the description of the the group of soft braided tensor autoequivalences of the Drinfeld centre $\mathcal{Z}(G)$ (section 2.4). Examples of non-trivial soft braided tensor autoequivalences are given in section 3 . We conclude with a sketch of a construction of non-trivial conformal field theories with charge conjugation modular invariant (section 4).

Throughout $k$ denote an algebraically closed field of characteristic zero. We will work with tensor categories. A category $\mathcal{C}$ is tensor over $k$ if it is monoidal and enriched in the category Vect $_{k}$ of finite dimensional vector spaces over $k$. That is hom-sets $\mathcal{C}(X, Y)$ for $X, Y \in \mathcal{C}$ are finite dimensional vector spaces over $k$ and the composition and tensor product of morphisms are bilinear maps.

A tensor category is fusion if it is semi-simple with finitely many simple objects. Note that for a semi-simple $\mathcal{C}$ the natural embedding of the Grothendieck group into the dual of the endomorphism algebra of the identity functor

$$
K_{0}(\mathcal{C}) \rightarrow \operatorname{End}\left(I d_{\mathcal{C}}\right)^{*}, \quad X \mapsto\left(a \mapsto a_{X}\right), \quad X \in \mathcal{C}, a \in \operatorname{End}\left(I d_{\mathcal{C}}\right)
$$

induces an isomorphism

$$
K_{0}(\mathcal{C}) \otimes_{\mathbb{Z}} k \rightarrow \operatorname{End}\left(I d_{\mathcal{C}}\right)
$$

\section{Acknowledgment}

The author would like to thank D. Nikshych, V. Ostrik and C. Schweigert for very useful comments on the preliminary version of the paper.

\section{Group-theoretical braided fusion categories and their au- toequivalences}

\subsection{Soft tensor autoequivalences}

Let $\mathcal{C}$ be a tensor category. A tensor autoequivalence $F: \mathcal{C} \rightarrow \mathcal{C}$ is called $s o f t$ if it is isomorphic as just a $k$-linear functor to the identity functor $I d_{\mathcal{C}}$.

Remark 2.1. Any soft tensor autoequivalence $F: \mathcal{C} \rightarrow \mathcal{C}$ is a twisted form of the identity functor $I d_{\mathcal{C}}$ in the terminology of [6]. That is $F$ is tensor isomorphic to the identity functor $I d_{\mathcal{C}}$ with the tensor structure given by a collection of automorphisms $\gamma_{X, Y}: X \otimes Y \rightarrow X \otimes Y$ natural in $X, Y \in \mathcal{C}$ and satisfying the normalised 2-cocycle condition (see [6] for details).

For a tensor category $\mathcal{C}$ denote by $A u t_{\otimes}(\mathcal{C})$ the group of tensor isomorphism classes of tensor autoequivalences of $\mathcal{C}$. Denote by $A u t_{\otimes}^{1}(\mathcal{C})$ the subgroup of $A u t_{\otimes}(\mathcal{C})$ consisting of isomorphism classes of soft tensor autoequivalences. Clearly the subgroup $A u t_{\otimes}^{1}(\mathcal{C}) \subset A u t_{\otimes}(\mathcal{C})$ is normal, since it is the kernel of the homomorphism forgetting tensor structure

$$
\operatorname{Aut}_{\otimes}(\mathcal{C}) \rightarrow \operatorname{Aut}(\mathcal{C})
$$

Here $A u t(\mathcal{C})$ is the group of isomorphism classes of $k$-linear autoequivalences of $\mathcal{C}$.

Remark 2.2. Note that for a fusion category $\mathcal{C}$ a tensor autoequivalence $F: \mathcal{C} \rightarrow \mathcal{C}$ is soft if and only if it induces a trivial automorphism of the Grothendieck ring $K_{0}(F)=1: K_{0}(\mathcal{C}) \rightarrow K_{0}(\mathcal{C})$. 
In other words $A u t_{\otimes}^{1}(\mathcal{C})$ is the kernel of the natural action of $A u t_{\otimes}(\mathcal{C})$ on the Grothendieck ring $K_{0}(\mathcal{C})$ :

$$
A u t_{\otimes}^{1}(\mathcal{C})=\operatorname{ker}\left(A u t_{\otimes}(\mathcal{C}) \rightarrow A u t\left(K_{0}(\mathcal{C})\right)\right) .
$$

Due to the isomorphism (11) $A u t_{\otimes}^{1}(\mathcal{C})$ is also the kernel of the natural action of $A u t_{\otimes}(\mathcal{C})$ on the endomorphism algebra of the identity functor

$$
A u t_{\otimes}^{1}(\mathcal{C})=\operatorname{ker}\left(A u t_{\otimes}(\mathcal{C}) \rightarrow \operatorname{Aut}\left(\operatorname{End}\left(\operatorname{Id}_{\mathcal{C}}\right)\right)\right) .
$$

Similarly for a braided tensor category $\mathcal{C}$ denote by $A u t_{b r}(\mathcal{C})$ the group of tensor isomorphism classes of braided tensor autoequivalences of $\mathcal{C}$. Denote by $A u t_{b r}^{1}(\mathcal{C})$ the normal subgroup of $A u t_{b r}(\mathcal{C})$ consisting of isomorphism classes of soft braided tensor autoequivalences.

Remark 2.3. Similarly to the remark 2.2 for fusion $\mathcal{C}$ the group $A u t_{b r}^{1}(\mathcal{C})$ is the kernel of the natural action of $A u t_{b r}(\mathcal{C})$ on the Grothendieck ring $K_{0}(\mathcal{C})$ :

$$
A u t_{b r}^{1}(\mathcal{C})=\operatorname{ker}\left(\operatorname{Aut}_{b r}(\mathcal{C}) \rightarrow \operatorname{Aut}\left(K_{0}(\mathcal{C})\right)\right)
$$

and on the endomorphism algebra of the identity functor:

$$
A u t_{b r}^{1}(\mathcal{C})=\operatorname{ker}\left(A u t_{b r}(\mathcal{C}) \rightarrow \operatorname{Aut}\left(\operatorname{End}\left(\operatorname{Id}_{\mathcal{C}}\right)\right)\right) .
$$

We say that braided tensor autoequivalence $F: \mathcal{C} \rightarrow \mathcal{C}$ preserves a full tensor subcategory $\mathcal{D} \subset \mathcal{C}$ if there is a braided tensor autoequivalence $\left.F\right|_{\mathcal{D}}: \mathcal{D} \rightarrow \mathcal{D}$ such that the diagram of functors

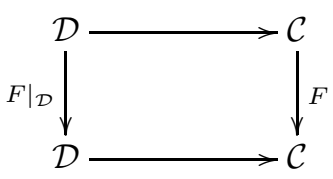

commutes up to a braided natural isomorphism.

The following is straightforward.

Lemma 2.4. Let $\mathcal{D}$ be a full tensor subcategory of a braided tensor category $\mathcal{C}$. Then any soft braided tensor autoequivalence $F$ of $\mathcal{C}$ preserves $\mathcal{D}$.

In particular there is defined a homomorphism of groups

$$
A u t_{b r}^{1}(\mathcal{C}) \rightarrow A u t_{b r}^{1}(\mathcal{D}),\left.\quad F \mapsto F\right|_{\mathcal{D}} .
$$

\subsection{Soft tensor autoequivalences of graded vector spaces}

Here we recall a well-known description of tensor autoequivalences for categories of graded vector spaces.

Let $G$ be a group. A $G$-graded vector space $V$ is a direct sum $\oplus_{g \in G} V_{g}$ of vector spaces labelled by elements of $G$. A morphism of $G$-graded vector spaces $U \rightarrow V$ is the direct sum $\oplus_{g \in G} f_{g}$ of homomorphisms $f_{g}: U_{g} \rightarrow V_{g}$.

Denote by $\mathcal{V}(G)$ the category of finite dimensional $G$-graded vector spaces.

Define the $G$-grading on the tensor product $U \otimes V$ of $G$-graded vector spaces:

$$
(U \otimes V)_{g}=\bigoplus_{g_{1} g_{2}=g} U_{g_{1}} \otimes V_{g_{2}} .
$$

Denote by $I \in \mathcal{V}(G)$ the one-dimensional vector space concentrated in the trivial degree

$$
I_{e}=k, \quad I_{g}=0, \quad g \neq e .
$$

The category $\mathcal{V}(G)$ is tensor. 
More generally for $f \in G$ denote by $I(f) \in \mathcal{V}(G)$ the one-dimensional vector space concentrated in the trivial degree

$$
I(f)_{f}=k, \quad I_{g}=0, \quad g \neq f .
$$

Clearly $I(f)$ is a simple object of $\mathcal{V}(G)$. The category $\mathcal{V}(G)$ is fusion and any simple object of $\mathcal{V}(G)$ is isomorphic to $I(f)$ for some $f \in G$. Tensor product of simple objects has the form

$$
I(f) \otimes I(g) \simeq I(f g) .
$$

The Grothendieck ring of $\mathcal{V}(G)$ is the group ring of $G$ :

$$
K_{0}(\mathcal{V}(G)) \simeq \mathbb{Z}[G] .
$$

A tensor autoequivalence $F$ of $\mathcal{V}(G)$ induces an automorphism of the group of isomorphism classes of simple objects. Hence we have a homomorphism

$$
A u t_{\otimes}(\mathcal{V}(G)) \rightarrow \operatorname{Aut}(G) .
$$

Let $\phi: G \rightarrow F$ be a homomorphism of groups. For an $F$-graded vector space $V=\oplus_{f \in F} V_{f}$ define the $G$-grading on $V$ by $V_{g}=V_{\phi(g)}$. We call the $G$-graded vector space $\phi^{*}(V)=\oplus_{g \in G} V_{g}$ the inverse image of $V$ along the homomorphism $\phi: G \rightarrow F$. The inverse image functor

$$
\phi^{*}: \mathcal{V}(F) \rightarrow \mathcal{V}(G) \quad V \mapsto \phi^{*}(V)
$$

along a group homomorphism $\phi: G \rightarrow F$ is tensor. Clearly $\phi^{*} \circ \psi^{*}=(\psi \phi)^{*}$ for group homomorphisms $\phi: G \rightarrow H$ and $\psi: H \rightarrow F$. In particular group automorphisms of $G$ give rise to tensor autoequivalences of $\mathcal{V}(G)$. Thus we have a homomorphism of groups

$$
\operatorname{Aut}(G) \rightarrow \operatorname{Aut}_{\otimes}(\mathcal{V}(G)) \quad \phi \mapsto\left(\phi^{-1}\right)^{*}
$$

Clearly the effect of $\phi^{*}$ on isomorphism classes of simple objects is $\phi^{-1}$ :

$$
\phi^{*}(I(f))=I\left(\phi^{-1}(f)\right) .
$$

Thus the homomorphism (2.5) is a splitting for the epimorphism (3). In other words $A u t_{\otimes}(\mathcal{V}(G))$ is the semi-direct product of $\operatorname{Aut}(G)$ and the kernel of (3).

Now we describe the kernel of (3). By the remark 2.2 the kernel coincides with the group $\operatorname{Aut}_{\otimes}^{1}(\mathcal{V}(G))$ of soft tensor autoequivalences of $\mathcal{V}(G)$. By the remark 2.1 a soft tensor autoequivalence of $\mathcal{V}(G)$ is a twisted form of the identity functor. Let $\gamma: G^{\times 2} \rightarrow k^{*}$ be a function. Here $k^{*}=k \backslash\{0\}$ is the multiplicative group of the ground field $k$. For $U, V \in \mathcal{V}(G)$ define an isomorphism

$$
I d(\gamma)_{U, V}: U \otimes V \rightarrow U \otimes V, \quad u \otimes v \mapsto \gamma(f, g)(u \otimes v), \quad u \in U_{f}, v \in V_{g} .
$$

Clearly the collection $I d(\gamma)_{U, V}$ is natural in $U, V \in \mathcal{V}(G)$. Moreover it is the most general form of a natural collection of isomorphisms $U \otimes V \rightarrow U \otimes V$.

Consider $I d(\gamma)_{U, V}$ as the tensor structure for the identity functor $I d: \mathcal{V}(G) \rightarrow \mathcal{V}(G)$. The coherence axiom for the tensor structure of $I d(\gamma)_{U, V}$ is equivalent to the equation

$$
\gamma(f, g h) \gamma(g, h)=\gamma(f g, h) \gamma(f, g)
$$

for any $f, g, h \in G$. Thus $\gamma$ is a 2-cocycle of $G$ with coefficients in $k^{*}$. Unit axioms are equivalent to the normalisation condition:

$$
\gamma(f, e)=\gamma(e, g)=1 \quad f, g \in G .
$$

Thus $\operatorname{Id}(\gamma)$ with $\gamma \in Z^{2}\left(G, k^{*}\right)$ is a (general) twisted form of the identity functor. The composition of twisted forms corresponds to the product of cocycles:

$$
\operatorname{Id}(\gamma) \circ \operatorname{Id}\left(\gamma^{\prime}\right)=\operatorname{Id}\left(\gamma \gamma^{\prime}\right) \text {. }
$$


A natural transformation $I d \rightarrow I d$ amounts to a function $c: G \rightarrow k^{*}$ :

$$
c_{V}: V \rightarrow V, \quad v \mapsto c(g) v, \quad v \in V_{g} .
$$

A natural transformation $\operatorname{Id}(\gamma) \rightarrow \operatorname{Id}\left(\gamma^{\prime}\right)$ is tensor if the function $c: G \rightarrow k^{*}$ is a coboundary for $\gamma\left(\gamma^{\prime}\right)^{-1}$ :

$$
\gamma(f, g) c(f g)=c(f) c(g) \gamma^{\prime}(f, g) .
$$

Thus we have the following.

Proposition 2.5. The group of isomorphism classes of tensor autoequivalences of the category $\mathcal{V}(G)$ is the semi-direct product:

$$
A u t_{\otimes}(\mathcal{V}(G)) \simeq \operatorname{Aut}(G) \ltimes H^{2}\left(G, k^{*}\right) .
$$

In particular the group of soft tensor autoequivalences of $\mathcal{V}(G)$ is the Schur multiplier of $G$ :

$$
A u t_{\otimes}^{1}(\mathcal{V}(G)) \simeq H^{2}\left(G, k^{*}\right) .
$$

Remark 2.6. With a invertible object $P$ in a tensor category $\mathcal{C}$ one can associate a tensor autoequivalence

$$
P \otimes \otimes P^{-1}: \mathcal{C} \rightarrow \mathcal{C}, \quad X \mapsto P \otimes X \otimes P
$$

the inner autoequivalence corresponding to $P \in \mathcal{C}$. The quotient group of $A u t_{\otimes}(\mathcal{C})$ by inner autoequivalences is denoted by $\mathrm{Out}_{\otimes}(\mathcal{C})$.

Clearly the inner tensor autoequivalence $I(g) \otimes \otimes I\left(g^{-1}\right)$ corresponding to the invertible object $I(g) \in \mathcal{V}(G)$ coincides with the inverse image $\phi_{g}^{*}$ along the inner automorphism $\phi_{g}(x)=g^{-1} x g$. This give a categorical action (the adjoint action) of the group $G$ (the group of isomorphism classes of invertible simple objects) on the category $\mathcal{V}(G)$. The group of outer tensor autoequivalences of

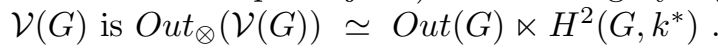

\subsection{Soft braided tensor autoequivalences of categories of representa- tions}

For a finite group $G$ denote by $\mathcal{R} e p(G)$ the category of finite dimensional representations. The category $\mathcal{R} \operatorname{ep}(G)$ is a braided (symmetric) tensor category. Representation categories are contravariant in $G$ : for a group homomorphism $\phi: G \rightarrow F$ there is a braided tensor functor

$$
\phi^{*}: \mathcal{R} e p(F) \rightarrow \mathcal{R} e p(G)
$$

called the inverse image along $\phi$. More precisely for an $F$ representation $V$ the $G$-action on $\phi^{*}(V)$ has the form $g(v)=\phi(g)(v)$, where $g \in G$ and $v \in V$. Clearly $\phi^{*} \circ \psi^{*}=(\psi \phi)^{*}$ for group homomorphisms $\phi: G \rightarrow H$ and $\psi: H \rightarrow F$. In particular group automorphisms of $G$ give rise to braided tensor autoequivalences of $\mathcal{R} e p(G)$. Inner automorphisms (automorphisms of the form $\phi(x)=g x g^{-1}$ ) are tensor isomorphic to the identity functor. Thus we have a homomorphism of groups

$$
\operatorname{Out}(G) \rightarrow \operatorname{Aut}_{b r}(\mathcal{R} e p(G)) \quad \phi \mapsto\left(\phi^{-1}\right)^{*}
$$

Here $\operatorname{Out}(G)$ is the group of outer automorphisms of $G$, that is the quotient of the group of automorphisms $\operatorname{Aut}(G)$ by its normal subgroup $\operatorname{Inn}(G)$ consisting of inner automorphisms.

Proposition 2.7. The map (2.7) induces an isomorphism

$$
\operatorname{Aut}_{b r}(\mathcal{R e p}(G)) \simeq \operatorname{Out}(G)
$$

Proof. It follows form the Deligne's theorem (on the existence of a fibre functor) [11. 
Remark 2.8. The results of [7] provide a more elementary proof. It was proved in [7] that tensor autoequivalences of $\mathcal{R} e p(G)$ correspond to $G$-biGalois algebras. It is not hard to see that braided tensor autoequivalences of $\mathcal{R} \operatorname{ep}(G)$ correspond to commutative $G$-biGalois algebras. Being semisimple these algebras have to be isomorphic to the function algebra $k(G)$ with the left and right $G$-actions given by

$$
(g a)(x)=a(x g), \quad(a g)(x)=a(\phi(g) x), \quad g, x \in G, a \in k(G),
$$

where $\phi: G \rightarrow G$ is an isomorphism.

A group automorphism $\phi: G \rightarrow G$ is class-preserving if it preserves conjugacy classes of $G$, that is for any $x \in G$ there is $g \in G$ such that $\phi(x)=g x g^{-1}$. It is straightforward that classpreserving automorphisms are closed under the composition. Denote by $A u t_{c l}(G)$ the group of class-preserving automorphisms of $G$. Clearly inner automorphisms are class-preserving. The quotient $\operatorname{Aut}_{c l}(G) / \operatorname{Inn}(G)$ is denoted $O u t_{c l}(G)$.

Proposition 2.9. The group of isomorphism classes of soft braided tensor autoequivalences of the category $\mathcal{R} \operatorname{ep}(G)$ is isomorphic to the group of outer class-preserving automorphisms of $G$ :

$$
A u t_{b r}^{1}(\mathcal{R e p}(G)) \simeq \operatorname{Out}_{c l}(G) .
$$

Proof. By the remark 2.3 it is enough to prove that $O u t_{c l}(G)$ is the kernel of the natural action of $\operatorname{Out}(G)$ on the Grothendieck ring $K_{0}(\mathcal{R} e p(G))$. It is well known (from the character theory) that $K_{0}(\mathcal{R} e p(G))$ is naturally isomorphic to the character ring $R(G)$. Moreover the character ring $R(G)$ is a subring of the algebra $R(G) \otimes_{\mathbb{Z}} k$ and the algebra $R(G) \otimes_{\mathbb{Z}} k$ coincides with the algebra of $k$-valued class functions (functions constant on conjugacy classes of $G$ ). Clearly the kernel of the natural action of $O u t(G)$ on class functions is $O u t_{c l}(G)$.

\subsection{Soft braided tensor autoequivalences of Drinfeld centres of finite groups}

We call a $G$-action on a $G$-graded vector space $V=\oplus_{g \in G} V_{g}$ compatible (with the grading) if $f\left(V_{g}\right)=V_{f g f^{-1}}$. Let $\mathcal{Z}(G)$ be the category $G$-graded vector spaces with compatible $G$-actions and with morphism being linear maps preserving grading and action. Define the tensor product $V \otimes U$ of objects $V, U \in \mathcal{Z}(G)$ as the tensor product of $G$-graded vector spaces with the charge conjugation $G$-action. The category $\mathcal{Z}(G)$ is a tensor category with respect to this tensor product and the trivial associativity constraint. Moreover $\mathcal{Z}(G)$ is braided with the braiding

$$
c_{V, U}(v \otimes u)=f(v) \otimes u, \quad v \in V_{f}, u \in U .
$$

We call the category $\mathcal{Z}(G)$ the Drinfeld centre of $G$ (the author first learned about Drinfeld's work on these categories from [32]).

The functor

$$
\mathcal{R} e p(G) \rightarrow \mathcal{Z}(G)
$$

considering a $G$-representation as the trivially $G$-graded (concentrated in the trivial degree) is a braided tensor fully faithful functor (full embedding). This functor has a tensor splitting, more precisely the functor

$$
\mathcal{Z}(G) \rightarrow \mathcal{R} e p(G)
$$

forgetting $G$-grading is tensor and the composition $\mathcal{R} e p(G) \rightarrow \mathcal{Z}(G) \rightarrow \mathcal{R} e p(G)$ is the identity. The functor forgetting $G$-action

$$
\mathcal{Z}(G) \rightarrow \mathcal{V}(G)
$$

is also tensor.

Recall that the monoidal centre $\mathcal{Z}(\mathcal{V}(G))$ of the category of graded vector spaces is braided equivalent to $\mathcal{Z}(G)$. Indeed an object $Z \in \mathcal{Z}(\mathcal{V}(G))$ has a natural half-braiding in $U \in \mathcal{V}(G)$ :

$$
z_{U}: U \otimes Z \rightarrow Z \otimes U, \quad z_{U}(u \otimes z)=f(z) \otimes u, \quad u \in U_{f}, z \in Z
$$


and the braided tensor functor

$$
\mathcal{Z}(G) \rightarrow \mathcal{Z}(\mathcal{V}(G)), \quad Z \mapsto(Z, z)
$$

is an equivalence (see e.g. [7 for details). By the functoriality of the monoidal centre there is a homomorphism

$$
A u t_{\otimes}(\mathcal{V}(G)) \rightarrow \operatorname{Aut}_{b r}(\mathcal{Z}(G)) \quad F \mapsto \tilde{F} .
$$

Here $\tilde{F}: \mathcal{Z}(G) \rightarrow \mathcal{Z}(G)$ is the braided tensor functor associated with a tensor functor $F: \mathcal{V}(G) \rightarrow$ $\mathcal{V}(G)$ as follows $\tilde{F}(Z, z)=(F(Z), F(z))$, where $F(z)_{U}$ is given by

$$
F(U) \otimes F(Z) \stackrel{F_{U, Z}}{\longrightarrow} F(U \otimes Z) \stackrel{F\left(z_{U}\right)}{\longrightarrow} F(Z \otimes U) \stackrel{F_{Z, U}^{-1}}{\longrightarrow} F(Z) \otimes F(U)
$$

The tensor structure of $\tilde{F}$ is the tensor structure of $F: \tilde{F}_{(Z, z),\left(Z^{\prime}, z^{\prime}\right)}=F_{Z, Z^{\prime}}$.

Thus there is a homomorphism of groups

$$
\operatorname{Aut}(G) \ltimes H^{2}\left(G, k^{*}\right) \simeq \operatorname{Aut}_{\otimes}(\mathcal{V}(G)) \rightarrow \operatorname{Aut}_{b r}(\mathcal{Z}(G))
$$

We can describe braided autoequivalences $\tilde{F}_{\phi, \gamma}: \mathcal{Z}(G) \rightarrow \mathcal{Z}(G)$ explicitly. First note that for a group isomorphism $\phi: G \rightarrow F$ there is a braided tensor equivalence

$$
\phi^{*}: \mathcal{Z}(F) \rightarrow \mathcal{Z}(G)
$$

called the inverse image along $\phi$. For $V \in \mathcal{Z}(F)$ the $G$-action on $\phi^{*}(V)$ has the form $g(v)=$ $\phi(g)(v)$, where $g \in G$ and $v \in V$ and the $G$-grading is defined by $V_{g}=V_{\phi^{-1}(g)}$. Clearly $\phi^{*} \circ \psi^{*}=$ $(\psi \phi)^{*}$ for group isomorphisms $\phi: G \rightarrow H$ and $\psi: H \rightarrow F$. This gives the homomorphism

$$
\operatorname{Aut}(G) \rightarrow \operatorname{Aut}_{b r}(\mathcal{Z}(G)) .
$$

It is easy to see that the homomorphism (9) factors through the outer automorphism group $\operatorname{Out}(G)$.

For $\gamma \in Z^{2}\left(G, k^{*}\right)$ the tensor autoequivalence $F_{\gamma}: \mathcal{V}(G) \rightarrow \mathcal{V}(G)$ induces a braided tensor autoequivalence $\tilde{F}_{\gamma}: \mathcal{Z}(G) \rightarrow \mathcal{Z}(G)$. The autoequivalence $\tilde{F}_{\gamma}$ does not change the $G$-grading of $V \in \mathcal{Z}(G)$ but changes the $G$-action to

$$
f * v=\frac{\gamma(f, g)}{\gamma(g, f)} f(v), \quad v \in V_{g}
$$

The braided autoequivalence $\tilde{F}_{\phi, \gamma}: \mathcal{Z}(G) \rightarrow \mathcal{Z}(G)$ is the composition $F_{\gamma} \circ \phi^{*}$.

The monoidal centre $\mathcal{Z}(\mathcal{R} e p(G))$ of the representation category is also braided equivalent to $\mathcal{Z}(G)$. Again an object $Z \in \mathcal{Z}(\mathcal{V}(G))$ has an (inverse) half-braiding natural in $U \in \mathcal{R} e p(G)$ :

$$
z_{U}: Z \otimes U \rightarrow U \otimes Z, \quad z_{U}(z \otimes u)=f(u) \otimes z, \quad u \in U, z \in Z_{f} .
$$

Similarly we have a homomorphism

$$
A u t_{\otimes}(\mathcal{R} e p(G)) \rightarrow \operatorname{Aut}_{b r}(\mathcal{Z}(G)) .
$$

In particular we have a homomorphism $\operatorname{Out}(G) \simeq \operatorname{Aut}_{b r}(\mathcal{R} e p(G)) \rightarrow \operatorname{Aut}_{b r}(\mathcal{Z}(G))$ which coincides with (the factorisation of) (91).

The following statement was proved in [37, Corollary 6.9] and characterises the image of (8).

Proposition 2.10. The group $\operatorname{Aut}_{b r}(\mathcal{Z}(G), \mathcal{R} e p(G))$ of the group Aut $t_{b r}(\mathcal{Z}(G))$ consisting of tensor isomorphism classes of braided tensor autoequivalences of $\mathcal{Z}(G)$ preserving the subcategory $\mathcal{R e p}(G) \rightarrow \mathcal{Z}(G)$ is $\operatorname{Out}(G) \ltimes H^{2}\left(G, k^{*}\right)$.

That is braided tensor autoequivalence of $\mathcal{Z}(G)$ preserving the subcategory $\mathcal{R} e p(G) \rightarrow \mathcal{Z}(G)$ has the form $\tilde{F}_{\phi, \gamma}$ for a group automorphism $\phi: G \rightarrow G$ and a 2-cocycle $\gamma \in Z^{2}\left(G, k^{*}\right)$. 
Remark 2.11. Here we briefly recall the character theory of the category $\mathcal{Z}(G)$. We use slightly different notation comparing e.g. to [13. The character of an object $V$ of $\mathcal{Z}(G)$ is a function $\chi_{V}:\left\{(f, g) \in G^{\times 2} \mid f g=g f\right\} \rightarrow k$ defined by

$$
\chi_{V}(f, g)=\operatorname{tr}_{V_{f}}(g)
$$

where $\operatorname{tr}_{V_{f}}(g)$ is the trace of the linear operator $g: V_{f} \rightarrow V_{f}, \quad v \mapsto g(v)$. It is straightforward that a character is a double class function, that is

$$
\chi\left(h f h^{-1}, h g h^{-1}\right)=\chi(f, g), \quad \forall h \in G .
$$

The character of the tensor product $V \otimes U$ has the following expression in terms of the characters of $V, U$ :

$$
\chi_{U \otimes V}(f, g)=\sum_{f_{1} f_{2}=f} \chi_{U}\left(f_{1}, g\right) \chi_{V}\left(f_{2}, g\right)
$$

where the sum is taken over all elements $f_{1}, f_{2}$ in the centraliser $C_{G}(g)$.

Here we have the main result of the paper describing soft braided tensor autoequivalences of $\mathcal{Z}(G)$.

Theorem 2.12. The group $A u t_{b r}^{1}(\mathcal{Z}(G))$ of tensor isomorphism classes of soft braided tensor autoequivalences of $\mathcal{Z}(G)$ is the subgroup of the semi-direct product Out $(G) \ltimes H^{2}\left(G, k^{*}\right)$ consisting of such pairs $(\phi, \gamma)$ that

$$
\chi(\phi(f), \phi(g))=\frac{\gamma(f, g)}{\gamma(g, f)} \chi(f, g), \quad f, g \in G
$$

for all double class functions $\chi$.

Proof. By lemma 2.4 a soft braided tensor autoequivalence $F$ of $\mathcal{Z}(G)$ preserves the subcategory $\mathcal{R e p}(G) \rightarrow \mathcal{Z}(G)$. By proposition 2.10 $F$ is tensor isomorphic to $\tilde{F}_{\phi, \gamma}$ for a group automorphism $\phi: G \rightarrow G$ and a 2-cocycle $\gamma \in Z^{2}\left(G, k^{*}\right)$.

By the remark 2.3 $F: \mathcal{Z}(G) \rightarrow \mathcal{Z}(G)$ is soft if and only if acts trivially on the Grothendieck ring $K_{0}(\mathcal{Z}(G))$. According to the remark $2.11 K_{0}(\mathcal{Z}(G))$ embeds in the algebra $K_{0}(\mathcal{Z}(G)) \otimes_{\mathbb{Z}} k$ and the algebra $K_{0}(\mathcal{Z}(G)) \otimes_{\mathbb{Z}} k$ coincides with the algebra of $k$-valued double class functions. We have the following formula for the character of $\tilde{F}_{\phi, \gamma}(V)$ :

$$
\chi_{\tilde{F}_{\phi, \gamma}(V)}(f, g)=\frac{\gamma(f, g)}{\gamma(g, f)} \chi_{V}(\phi(f), \phi(g)) .
$$

Thus $\tilde{F}_{\phi, \gamma}$ is soft if and only if the pair $\phi, \gamma$ satisfies the condition (10).

We call a group automorphism $\phi: G \rightarrow G$ doubly class-preserving if it preserves conjugacy classes of commuting pairs of elements of $G$, that is for any $x, y \in G$ such that $x y=y x$ there is $g \in G$ such that $\phi(x)=g x g^{-1}, \phi(y)=g y g^{-1}$. It is straightforward that doubly class-preserving automorphisms are closed under the composition. Denote by $A u t_{2-c l}(G)$ the group of doubly class-preserving automorphisms of $G$. Clearly inner automorphisms are doubly class-preserving. The quotient $A u t_{2-c l}(G) / \operatorname{Inn}(G)$ is denoted Out $_{2-c l}(G)$.

Thus $\tilde{F}_{\gamma}: \mathcal{Z}(G) \rightarrow \mathcal{Z}(G)$ is soft if and only if

$$
\frac{\gamma(f, g)}{\gamma(g, f)}=1 \quad \text { for any commuting } f, g \in G .
$$

Denote by $B(G)$ the subgroup of $H^{2}\left(G, k^{*}\right)$ consisting of classes of $\gamma$ satisfying (11).

Corollary 2.13. The semi-direct product Out $t_{2-c l}(G) \ltimes B(G)$ is a subgroup of the group Aut $t_{b r}^{1}(\mathcal{Z}(G))$ of tensor isomorphism classes of soft braided tensor autoequivalences of $\mathcal{Z}(G)$. 
Corollary 2.14. The group $A u t_{b r}^{1}(\mathcal{Z}(G))$ of tensor isomorphism classes of soft braided tensor autoequivalences of $\mathcal{Z}(G)$ fits into an exact sequence

$$
1 \longrightarrow B(G) \longrightarrow A u t_{b r}^{1}(\mathcal{Z}(G)) \longrightarrow O u t_{c l}(G)
$$

Proof. The image of the homomorphism $A u t_{b r}^{1}(\mathcal{Z}(G)) \rightarrow$ Out $(G)$ restricting to the subcategory $\mathcal{R} e p(G) \rightarrow \mathcal{Z}(G)$ is clearly in the group $A u t_{b r}^{1}(\mathcal{R} e p(G)) \simeq \operatorname{Out}_{c l}(G)$ of soft braided tensor autoequivalences of $\operatorname{Rep}(G)$ which is isomorphic to the group of outer automorphisms of $G$. The kernel of the restriction homomorphism consists of classes of $\tilde{F}_{\gamma}$ with $\gamma \in Z^{2}\left(G, k^{*}\right)$ satisfying the condition (11) with trivial $\phi$. That is $[\gamma] \in B(G)$.

Corollary 2.15. For a simple $G$ the group $\operatorname{Aut}_{b r}^{1}(\mathcal{Z}(G))$ is trivial.

\section{$3 \quad$ Examples}

Here we give examples of finite groups $G$ with non-trivial groups of soft braided autoequivalences $A u t_{b r}^{1}(\mathcal{Z}(G))$.

\subsection{Bogomolov multipliers}

It is well-known that for an abelian $A$ the map

$$
H^{2}\left(A, k^{*}\right) \rightarrow H o m\left(\Lambda^{2} A, k^{*}\right), \quad \gamma \mapsto\left(a \wedge b \mapsto \frac{\gamma(a, b)}{\gamma(b, a)}\right)
$$

is an isomorphism. Thus the group $B(G)$ can be described as the kernel

$$
\operatorname{ker}\left(H^{2}\left(G, k^{*}\right) \stackrel{r e s}{\longrightarrow} \bigoplus_{A \subset G} H^{2}\left(A, k^{*}\right)\right)
$$

of restriction homomorphisms, where the sum is taken over all (2-generated) abelian subgroups $A \subset G$.

It was proved in 2] that $B(G)$ coincides with the unramified Brauer group $H_{n r}^{2}\left(k(V)^{G}, k^{*}\right)$, where $V$ is a faithful representation of $G$ over $k$. Non-triviality of this group provides an obstruction to stable rationality of $k(V)^{G}$. The group (denoted $B_{0}(G)$ ) was called the Bogomolov multiplier of $G$ in [31. Recently quite a few examples of finite $G$ with no-trivial Bogomolov multiplier were presented in the literature. We copy some here.

Consider the group

$$
G=\left\langle a, b, c \mid a^{2}=b^{2}=1, c^{2}=[a, c], \quad[c, b]=[[c, a], a], \quad[[b, a], G]=1, \quad[G,[G,[G, G]]]\right\rangle .
$$

of class 3 and order 64 . It was proved in 34 that $B(G) \simeq \mathbb{Z} / 2 \mathbb{Z}$.

The following two groups [27] are metabelian (of class 2) of order $|G|=p^{7}$ and of period $p$.

$$
G=\left\langle a, b, c, d \mid a^{p}=b^{p}=c^{p}=d^{p}=1, \quad[a, b]=[c, d], \quad[b, d]=[a, b]^{\varepsilon}[a, c]^{\omega}, \quad[G,[G, G]]\right\rangle,
$$

where $\varepsilon=1$ for $p=2$ and $\varepsilon=0$ for odd primes $p$, and $\omega$ is a generator of the multiplicative group $(\mathbb{Z} / p \mathbb{Z})^{*}$.

The Bogomolov multiplier is $B(G) \simeq \mathbb{Z} / p \mathbb{Z} \times \mathbb{Z} / p \mathbb{Z}$.

$$
\left\langle a, b, c, d \mid a^{p}=b^{p}=c^{p}=d^{p}=1, \quad[a, b]=[c, d], \quad[a, c]=[a, d]=1, \quad[G,[G, G]]\right\rangle,
$$

The Bogomolov multiplier is $B(G) \simeq \mathbb{Z} / p \mathbb{Z}$. 


\subsection{Double class-preserving automorphisms}

First examples of non-inner double class-preserving automorphisms were given in [36]. Here we reproduce the construction along with some examples from [38.

Let $M$ be a (finite) abelian group. Consider the action of the additive group $\operatorname{End}(M)$ on $M \oplus M:$

$$
f(x, y)=(x, f(x)+y), \quad f \in \operatorname{End}(M), x, y \in M .
$$

Let $G=\operatorname{End}(M) \ltimes(M \oplus M)$ be the semi-direct product with respect to this action. Note that

$$
[G, G]=Z(G)=0 \ltimes(0 \oplus M) .
$$

In particular the group $G$ is metabelian.

For an additive subgroup $E \subset \operatorname{End}(M)$ consider the (normal) subgroup

$$
G(E)=E \ltimes(M \oplus M) \subset G .
$$

Consider

$$
\tilde{E}=\{g \in \operatorname{End}(M) \mid \forall x, y \in M \exists f \in E: \quad f(x)=g(x), f(y)=g(y)\} .
$$

It was argued in $\left[38\right.$, that the quotient $\tilde{E} / E$ embeds into $O u t_{2-c l}(G(E))$.

Now following 38 take $M$ to be an $n$-dimensional vector space over a finite field $\mathbb{F}_{q}$ and take $E=\mathfrak{s l}(M)=\{g \in \operatorname{End}(M) \mid \operatorname{tr}(g)=0\}$. It follows from double transitivity of the $\mathfrak{s l}(M)$-action on $M$ for $n \geq 3$ that $\widetilde{\mathfrak{s l}(M)}=\operatorname{End}(M)$.

Thus $\mathrm{Out}_{2-c l}(G(\mathfrak{s l}(M)))$ has an element of order $q$.

\section{Applications}

Here we sketch a construction of different conformal field theories with the same charge conjugation modular invariant.

\subsection{Lagrangian algebras of braided autoequivalences}

Let $F: \mathcal{C} \rightarrow \mathcal{C}$ be a braided tensor autoequivalence of a braided fusion category $\mathcal{C}$. Consider an object

$$
Z(F)=\bigoplus_{X \in \operatorname{Irr}(\mathcal{C})} X \otimes F(X)^{*} \in \mathcal{C} \otimes \overline{\mathcal{C}},
$$

where the sum is taken over isomorphism classes of simple objects of $\mathcal{C}$. Define a morphism $\mu: Z(F) \otimes Z(F) \rightarrow Z(F)$ in $\mathcal{C} \otimes \overline{\mathcal{C}}$ as the image of (the sum of) canonical elements under the map

$$
\begin{aligned}
& \bigoplus_{X, Y, Z \in \operatorname{Irr}(\mathcal{C})} \mathcal{C}(X \otimes Y, Z) \otimes_{k} \mathcal{C}(X \otimes Y, Z)^{*} \simeq \\
& \bigoplus_{X, Y, Z \in \operatorname{Irr}(\mathcal{C})} \mathcal{C}(X \otimes Y, Z) \otimes_{k} \mathcal{C}(Z, X \otimes Y) \simeq \\
& \bigoplus_{X, Y, Z \in \operatorname{Irr}(\mathcal{C})} \mathcal{C}(X \otimes Y, Z) \otimes_{k} \overline{\mathcal{C}}(F(Z), F(X \otimes Y)) \simeq \\
& \bigoplus_{X, Y, Z \in \operatorname{Irr}(\mathcal{C})} \mathcal{C}(X \otimes Y, Z) \otimes_{k} \overline{\mathcal{C}}(F(Z), F(Y) \otimes F(X))= \\
& \bigoplus_{X, Y, Z \in \operatorname{Irr}(\mathcal{C})} \mathcal{C}(X \otimes Y, Z) \otimes_{k} \overline{\mathcal{C}}\left(F(X)^{*} \otimes F(Y)^{*}, F(Z)^{*}\right)= \\
& \bigoplus_{X, Y, Z \in \operatorname{Irr}(\mathcal{C})}(\mathcal{C} \otimes \overline{\mathcal{C}})((X \otimes Y) \otimes(F(X) \otimes F(Y)), Z \otimes F(Z))= \\
& (\mathcal{C} \otimes \overline{\mathcal{C}})(Z(F) \otimes Z(F), Z(F))
\end{aligned}
$$


The first isomorphism comes from the non-degenerate pairing

$$
\mathcal{C}(X, W) \otimes_{k} \mathcal{C}(W, X) \rightarrow \mathcal{C}(X, X) \simeq k
$$

given by the composition of morphisms in $\mathcal{C}$ (here $X$ is simple); the second isomorphism is the effect on morphisms of the functor $F$; the third isomorphism is induced by the inverse to the composition

$$
F(Y) \otimes F(X) \stackrel{F_{Y, X}}{\longrightarrow} F(Y \otimes X) \stackrel{F\left(c_{Y, X}\right)}{\longrightarrow} F(X \otimes Y)
$$

Lemma 4.1. The pair $(Z(F), \mu)$ is a commutative algebra in $\mathcal{C} \otimes \overline{\mathcal{C}}$.

It follows from the results of [10, section 3.2] that $Z(F)$ is a Lagrangian algebra in $\mathcal{C} \otimes \overline{\mathcal{C}}$. Note that the algebras $Z(F), Z\left(F^{\prime}\right)$ are isomorphic if and only if the autoequivalences $F, F^{\prime}$ are tensor isomorphic.

For a Lagrangian algebra $Z \in \mathcal{C} \otimes \overline{\mathcal{C}}$ the class $[Z]$ in the Grothendieck ring $K_{0}(\mathcal{C} \otimes \overline{\mathcal{C}}) \simeq$ $K_{0}(\mathcal{C}) \times_{\mathbb{Z}} K_{0}(\mathcal{C})$ written in the basis of classes of simple objects of $\mathcal{C}$

$$
[Z]=\sum_{\chi, \xi} M_{\chi \xi} \chi \otimes \bar{\xi}^{*}
$$

gives rise to a non-negative integer matrix $M=\left(M_{\chi \xi}\right)$ called the modular invariant of $Z$.

We say that $Z$ has the charge conjugation modular invariant if the matrix $M$ is the identity $M_{\chi \xi}=\delta_{\chi \xi}$.

The next lemma follows from the results of [10, section 3.2].

Lemma 4.2. A Lagrangian algebra $Z \in \mathcal{C} \otimes \overline{\mathcal{C}}$ has the charge conjugation modular invariant if and only if $Z \simeq Z(F)$ for a soft braided tensor autoequivalence $F: \mathcal{C} \rightarrow \mathcal{C}$.

\subsection{Holomorphic permutation orbifolds}

Let $V$ be a holomorphic vertex operator algebra (for example $V=\mathfrak{e}_{8,1}$ ).

Let $G \subset S_{n}$ be a subgroup of the permutation group. The vertex operator subalgebra $\left(V^{\otimes n}\right)^{G}$ of invariants is called the chiral permutation orbifold of $V$.

According to [30] its category of representation is $\mathcal{R} e p\left(\left(V^{\otimes n}\right)^{G}\right)=\mathcal{Z}(G)$ (subject to the rationality of $\left.\left(V^{\otimes n}\right)^{G}\right)$.

According to [21, 26] a Lagrangian algebra $Z \in \mathcal{Z}(G) \otimes \overline{\mathcal{Z}}(G)$ gives rise to a rational conformal field theory with the left (right) chiral algebras $\left(V^{\otimes n}\right)^{G}$ and the modular invariant $[Z]$. In particular taking $Z=Z(F)$ for a soft braided tensor autoequivalence $F: \mathcal{Z}(G) \rightarrow \mathcal{Z}(G)$ provides an example of non-trivial rational conformal field theory with the charge conjugation modular invariant.

\section{References}

[1] K. Barron, C. Dong, G. Mason, Twisted sectors for tensor product vertex operator algebras associated to permutation groups, Commun. Math. Phys. 227 (2002), no. 2, 349-384.

[2] F. A. Bogomolov, The Brauer group of quotient spaces by linear group actions, Math. USSR Izv. 30 (1988), 455-485.

[3] R. Borcherds, Vertex algebras, Kac-Moody algebras, and the Monster. Proc. Nat. Acad. Sci. U.S.A. 83 (1986), no. 10, 30683071.

[4] A. Cappelli, C. Itzykson, J.-B. Zuber, The A-D-E classication of minimal and $A_{1}^{1}$ conformal invariant theories. Comm. Math. Phys. 113 (1987), no. 1, 1-26.

[5] H. Chu, S. Hu, M. Kang and B. Kunyavskii, Noethers problem and the unramied Brauer groups for groups of order 64. Intern. Math. Res. Notices 12 (2010), 2329-2366. 
[6] A. Davydov, Twisting of monoidal structures. Preprint of Max Planck Institut, MPI/95-123 (1995), arXiv:q-alg/9703001

[7] A. Davydov, Galois algebras and monoidal functors between categories of representation of finite groups. J.Algebra, 244 (2001), no. 1, 273-301.

[8] A. Davydov, Modular invariants for group-theoretic modular data I, J. Algebra, 323 (2010), pp. 1321-1348.

[9] A. Davydov, M. Müger, D. Nikshych, V. Ostrik, Witt group of non-degenerate braided fusion categories, Journal für die Reine und Angewandte Mathematik, 677 (2013), 135-177.

[10] A. Davydov, D. Nikshych, V. Ostrik, On the structure of the Witt group of braided fusion categories, Selecta Mathematica, (2013), 19, 1, 237-269.

[11] P. Deligne, Catégories tannakiennes. In The Grothendieck Festschrift, Volume 2, 111-195. Birkhauser, 1990.

[12] P. Di Francesco, P. Mathieu, and D. Sénéchal, Conformal Field Theory, Springer-Verlag, New York, 1997.

[13] R. Dijkgraaf, V. Pasquier, P. Roche. Quasi Hopf algebras, group cohomology and orbifold models. Nucl. Phys. B (Proc. Suppl.), 18B: 6072, 1990.

[14] V. Drinfeld, S. Gelaki, D. Nikshych, V. Ostrik, On braided fusion categories I, Selecta Math. 16 (2010), 1-119.

[15] J. Fjelstad, J. Fuchs, I. Runkel, and C. Schweigert, Uniqueness of open/ closed rational CFT with given algebra of open states, Adv. Theor. Math. Phys. 12 (2008), 1283-1375.

[16] I. Frenkel, Y. Huang, J. Lepowsky, On axiomatic approaches to vertex operator algebras and modules, Mem. Amer. Math. Soc., 104 (1993).

[17] J. Fuchs, I. Runkel, C. Schweigert, Conformal Correlation Functions, Frobenius Algebras and Triangulations Nucl. Phys. B 624 (2002). 452-468.

[18] J. Fuchs, I. Runkel, C. Schweigert, TFT construction of RCFT correlators I: Partition functions, Nucl.Phys. B 646 (2002), 353-497.

[19] J. Fuchs, I. Runkel, C. Schweigert, TFT construction of RCFT correlators II: Unoriented world sheets, Nucl. Phys. B 678 (2004), 511-637.

[20] J. Fuchs, I. Runkel, C. Schweigert, TFT construction of RCFT correlators III: Simple currents, Nucl.Phys. B 694 (2004), 277-353.

[21] J. Fuchs, I. Runkel, C. Schweigert, TFT construction of RCFT correlators IV: Structure constants and correlation functions. Nuclear Phys. B 715 (2005), no. 3, 539-638.

[22] J. Fuchs, I. Runkel, C. Schweigert, TFT construction of RCFT correlators V: Proof of modular invariance and factorisation, Theo. Appl. Cat., 16 (2006) 342-433.

[23] J. Fuchs, I. Runkel, C. Schweigert, Categorification and correlation functions in conformal field theory. Proceedings of ICM 2006.

[24] J. Fuchs, A. N. Schellekens, C. Schweigert, Galois Modular Invariants of WZW Models, Nucl. Phys. B437 (1995), 667-694.

[25] Y.-Z. Huang, Vertex operator algebras, fusion rules and modular transformations. Contemp. Math., 391 (2005), 135-148.

[26] Y.-Z. Huang, L. Kong, Full field algebras. Comm. Math. Phys. 272 (2007), no. 2, 345396. 
[27] U. Jezernik, P. Moravec, Universal commutator relations, Bogomolov multipliers, and commuting probability, arXiv:1307.6533.

[28] Kac, V., Vertex algebras for beginners. Second edition. University Lecture Series American Mathematical Society, Providence, RI, 1998.

[29] A. Kapustin, D. Orlov, Vertex Algebras, Mirror Symmetry, and D-Branes: The Case of Complex Tori, Commun. Math. Phys., 233 (2003), 79136.

[30] A. Kirillov Jr., Modular Categories and Orbifold Models, Commun. Math. Phys., 229 (2002), 309-335.

[31] B. E. Kunyavskii, The Bogomolov multiplier of nite simple groups, in Ratio- nality problems, pages 209217, edited by F. A. Bogomolov and Y. Tschinkel, Progress in Math. vol. 282, Birkhauser, Boston, 2010.

[32] A. Levin, seminar at Moscow University, c. 1990.

[33] G. Moore, N. Seiberg, Lectures on RCFT. Superstrings 89 (Trieste, 1989), 1129, World Sci. Publ.,1990.

[34] P. Moravec, Unramied Brauer groups of nite and innite groups, Amer. J. Math. 134 (2012), no. $6,1679-1704$.

[35] M. Müger, Conformal orbifold theories and braided crossed G-categories. Comm. Math. Phys. 260 (2005), no. 3, 727-762.

[36] B. Neumann, Not quite inner automorphisms, Bull. Austral. Math. Soc. 23 (1981), 461-469.

[37] D. Nikshych, B. Riepel, Categorical Lagrangian Grassmannians and Brauer-Picard groups of pointed fusion categories, arXiv:1309.5026.

[38] F. Szechtman, n-inner automorphisms of nite groups, Proc. Amer. Math. Soc. 131 (2003), 3657-3664.

[39] V. Turaev, Modular categories and 3-manifold invariants. Internat. J. Modern Phys. B 6 (1992), no. 11-12, 18071824. 